Maria Fernanda Furtado de Lima e Costa ${ }^{1, \text { II }}$

Sérgio Viana Peixoto"'I

Cibele Comini Césariv

Deborah Carvalho Maltav ${ }^{v, v I}$

Erly Catarina de Moura ${ }^{\mathrm{v}, \mathrm{VII}}$

Laboratório de Epidemiologia e Antropologia Médica. Centro de Pesquisas René Rachou. Fundação Oswaldo Cruz. Belo Horizonte, MG, Brasil

Departamento de Medicina Preventiva e Social. Faculdade de Medicina. Universidade Federal de Minas Gerais (UFMG). Belo Horizonte, MG, Brasi

III Departamento de Nutrição Clínica e Social. Escola de Nutrição. Universidade Federal de Ouro Preto. Ouro Preto, MG, Brasil

Departamento de Estatística. Instituto de Ciências Exatas. UFMG. Belo Horizonte, MG, Brasil

Coordenação de Doenças e Agravos Não Transmissíveis. Secretaria de Vigilância em Saúde. Ministério da Saúde. Brasília, DF, Brasil

Departamento de Enfermagem MaternoInfantil e Saúde Pública. Escola de Enfermagem. Universidade Federal de Minas Gerais. Belo Horizonte, MG, Brasil

VII Núcleo de Pesquisas Epidemiológicas em Nutrição e Saúde. Universidade de São Paulo. São Paulo, SP, Brasil

Correspondência | Correspondence: Maria Fernanda Furtado de Lima e Costa

Fundação Oswaldo Cruz

Centro de Pesquisas René Rachou

Av. Augusto de Lima, 1715

30190-002 Belo Horizonte, MG, Brasil

E-mail: lima-costa@cpqrr.fiocruz.br

\section{Comportamentos em saúde entre idosos hipertensos, Brasil, 2006}

\section{Health behaviors among older adults with hypertension, Brazil, 2006}

\section{RESUMO}

OBJETIVO: Estimar as prevalências de comportamentos prejudiciais à saúde e de outros fatores de risco cardiovascular entre idosos com hipertensão autoreferida e comparando-as com de não-hipertensos.

MÉTODOS: Foram utilizados dados do sistema de Vigilância de Fatores de Risco e Proteção para Doenças Crônicas por Inquérito Telefônico (VIGITEL), referentes aos 9.038 idosos residentes em domicílios com pelo menos uma linha telefônica fixa nas 26 capitais brasileiras e no Distrito Federal em 2006.

RESULTADOS: A prevalência de hipertensão auto-referida foi de 55\% (IC 95\%: 53;57). A maioria dos hipertensos apresentava concomitância de três ou mais fatores de risco $(69 \%$; IC 95\%: 67;71). Foram observadas altas prevalências de atividades físicas insuficientes no lazer (88\%; IC 95\%: 86;89) e do consumo de frutas e hortaliças inferior a cinco porções diárias $(90 \%$; IC 95\%: 88;90) entre hipertensos, seguidas pela adição de sal aos alimentos (60\%; IC 95\%: 57;63), consumo habitual de carnes gordurosas (23\%; IC 95\%: $21 ; 25)$, tabagismo (9\%; IC 95\%: 7;10) e consumo abusivo de álcool $(3 \%$; IC $95 \%: 2 ; 4)$. Essas prevalências foram semelhantes às observadas entre não hipertensos ( $p>0,05)$, exceto tabagismo. A prevalência do tabagismo foi menor entre hipertensos (razão de prevalência ajustada [RPA] $=0,75$; IC 95\%: $0,64 ; 0,89)$ e as prevalências de sobrepeso ( $\mathrm{RPA}=1,37$; IC $95 \%: 1,25 ; 1,49)$, dislipidemia (RPA 1,36; IC 95\%: 1,26;1,36) e diabetes (RPA=1,37; IC 95\%: $1,27 ; 1,37)$ foram mais altas.

CONCLUSÕES: Os resultados sugerem que, exceto tabagismo, os comportamentos prejudiciais à saúde entre idosos persistem após o diagnóstico da hipertensão arterial.

DESCRITORES: Idoso. Hipertensão, epidemiologia. Fatores de Risco. Doença Crônica, prevenção \& controle. Levantamentos Epidemiológicos. Brasil. Comportamentos em saúde. Entrevista por telefone. 


\section{ABSTRACT}

OBJECTIVE: To estimate the prevalence of unhealthy behaviors and other cardiovascular risk factors among older adults with self-reported arterial hypertension, and to compare these prevalences with those of nonhypertensives.

METHODS: Data used was obtained from the system Vigilância de Fatores de Risco e Proteção para Doenças Crônicas por Inquérito Telefônico (VIGITEL - Telephone-based Surveillance of Risk and Protective Factors for Chronic Diseases) referring to the 9,038 older adults living in households with at least one fixed telephone line in the 26 Brazilian state capitals and Federal District in 2006 .

RESULTS: Prevalence of self-reported hypertension was 55\% (95\% CI: 53;57). The majority of hypertensives showed three or more concomitant risk factors $(69 \% ; 95 \%$ CI: 67;71). It was found a high prevalence of insufficient physical activity during leisure (88\%; $95 \%$ CI: $86 ; 89)$ and of fruit and vegetable intake below five daily portions ( $90 \%$; $95 \%$ CI: 88;90) among hypertensive subjects, followed by adding salt to meals ( $60 \% ; 95 \%$ CI: $57 ; 63)$, regular intake of fatty meats (23\%; 95\% CI: $21 ; 25)$, smoking (9\%; 95\% CI: 7;10), and binge drinking $(3 \% ; 95 \%$ CI: 2;4). With the exception of smoking, these prevalences were similar to those reported by non-hypertensive subjects ( $p>0.05)$. Prevalence of smoking was lower among hypertensives (adjusted prevalence ratio [APR] $=$ $0.75 ; 95 \%$ CI: $0.64 ; 0.89$ ), whereas prevalence of overweight (APR $=1.37 ; 95 \%$ CI: $1.25 ; 1.49)$, dyslipidemia (APR $=1.36 ; 95 \%$ CI: $1.26 ; 1.36$ ), and diabetes $(\mathrm{APR}=1.37 ; 95 \% \mathrm{CI}: 1.27 ; 1.37)$ was higher.

CONCLUSIONS: Results suggest that, with the exception of smoking, unhealthy behaviors persist among older adults after hypertension is diagnosed.

DESCRIPTORS: Aged. Hypertension, epidemiology. Risk Factors. Chronic Disease, prevention \& control. Health Surveys. Brazil. Health behaviors. Telephone interview.

\section{INTRODUÇÃO}

A hipertensão arterial é o principal fator de risco para a mortalidade e a terceira causa mais importante de anos de vida com incapacidade no mundo. ${ }^{8}$ A sua prevalência global é de $26 \%$ na população adulta, com projeções crescentes nas próximas décadas. ${ }^{13} \mathrm{O}$ risco de desenvolver hipertensão arterial aumenta com a idade, sendo a doença crônica mais comum em idosos, com prevalência igual ou superior a $60 \%$ em países desenvolvidos, assim como na América Latina e Caribe. ${ }^{13}$

A hipertensão arterial não ocorre de forma isolada. A maioria dos hipertensos apresenta outros fatores de risco e a concomitância desses fatores aumenta o risco cardiovascular. ${ }^{12}$ Uma vez que muitos desses fatores são modificáveis, as recomendações para a prevenção e o controle da hipertensão arterial contemplam a abordagem dos múltiplos fatores de risco apresentados pelo indivíduo, incluindo os comportamentos em saúde. ${ }^{4,18}$
As estratégias voltadas para a população visando à promoção de comportamentos saudáveis têm por objetivo prevenir a hipertensão arterial. As estratégias voltadas para grupos de alto risco têm por objetivo reduzir a pressão arterial, controlar outros fatores de risco para eventos cardiovasculares e reduzir a dose dos medicamentos necessários para controlar a hipertensão. ${ }^{4,18}$ Existe consenso que tais estratégias sejam voltadas para cessação do tabagismo, redução do peso entre aqueles com sobrepeso, moderação no consumo de álcool, implementação de atividades físicas, redução do consumo de sal, aumento do consumo de frutas e hortaliças e a diminuição do consumo de alimentos gordurosos. ${ }^{18}$

Os comportamentos de saúde da população têm sido alvo de atenção crescente em diversos países, inclusive no Brasil. ${ }^{a}$ Entretanto, os comportamentos em saúde de idosos hipertensos têm recebido pouca atenção.

${ }^{a}$ Ministério da Saúde. Secretaria de Vigilância em Saúde. Secretaria de Atenção à Saúde. Política nacional de promoção da saúde. 2. ed, Brasília, DF; 2007 a. (Série B: Textos Básicos de Saúde. Série Pactos pela Saúde 2007, 7). 
O objetivo do presente estudo foi estimar as prevalências de comportamentos prejudiciais à saúde e de outros fatores de risco cardiovascular entre idosos com hipertensão auto-referida e comparando-as com de não-hipertensos.

\section{MÉTODOS}

Estudo transversal que utilizou dados do sistema de Vigilância de Fatores de Risco e Proteção para Doenças Crônicas por Inquérito Telefônico (VIGITEL). O VIGITEL baseia-se em amostra representativa de adultos residentes em domicílios servidos por pelo menos uma linha telefônica fixa nas capitais dos 26 estados brasileiros e no Distrito Federal. A amostragem consistiu no sorteio sistemático de 5.000 linhas telefônicas por cidade. Do total de linhas telefônicas sorteadas, 76.330 eram elegíveis para a pesquisa. Procedeu-se à enumeração dos residentes no domicílio com 18 ou mais anos de idade e, a seguir, ao sorteio de um dos residentes para ser entrevistado. Foram realizadas 54.360 entrevistas, correspondendo a 71,1\% dos domicílios selecionados. As entrevistas foram realizadas por telefone, entre agosto e dezembro de 2006. ${ }^{a}$ Foram incluídos na presente análise todos os 9.038 participantes com idade $\geq 60$ anos do inquérito telefônico realizado em 2006.

A variável dependente deste estudo foi a hipertensão arterial auto-referida, definida por meio de história de diagnóstico médico para a doença. As variáveis independentes do estudo incluíram idade, sexo, comportamentos em saúde, sobrepeso, diabetes mellitus auto-referido e dislipidemia auto-referida. Entre os comportamentos em saúde, foram considerados: tabagismo, consumo abusivo de bebidas alcoólicas, consumo insuficiente de frutas e hortaliças, consumo habitual de carnes com excesso de gordura, adição de sal à refeição pronta e atividade física insuficiente no lazer. A condição de fumante foi atribuída a todos que relataram fumar, independentemente da freqüência e intensidade do hábito. Consumo abusivo de bebidas alcoólicas foi definido como consumo de mais de quatro doses (mulheres) ou de mais de cinco doses (homens) em uma única ocasião nos 30 dias anteriores à entrevistas. Consumo insuficiente de frutas e hortaliças foi definido pela ingestão diária de menos de cinco porções desses alimentos pelo mesmo período. Foi considerado consumo de carnes com excesso de gordura a ingestão de carne vermelha gordurosa ou frango com pele, sem remover a gordura visível desses alimentos. O uso de sal foi definido como adição habitual ou esporádica da substância ao alimento pronto, no prato, exceto salada. A condição de atividade física insuficiente no lazer foi atribuída aos indivíduos que informaram não ter praticado atividades de intensidade leve ou moderada por pelo menos 30 min diários em cinco ou mais dias na semana ou atividades de intensidade vigorosa por pelo menos $20 \mathrm{~min}$ em três dias ou mais semana. Sobrepeso foi definido como índice de massa corporal (IMC - peso dividido pela altura ao quadrado) com valor igual ou superior a $25 \mathrm{~kg} / \mathrm{m}^{2}$, baseado na informação do peso e altura fornecidos pelo entrevistado. Maiores detalhes podem ser vistos em outra publicação. ${ }^{a}$

A análise dos dados foi baseada em razões de prevalência e intervalos com $95 \%$ de confiança estimadas por meio da regressão de Poisson. As razões de prevalência foram ajustadas por idade (variável contínua) ou ajustadas por idade e sexo, quando pertinente. A correlação tetracórica foi utilizada para examinar as correlações existentes entre diferentes comportamentos em saúde e sobrepeso. A ponderação para ajustar a distribuição sociodemográfica da amostra VIGITEL à distribuição da população adulta no Censo Demográfico de 2000 e o peso populacional de cada cidade foi incorporada à análise. ${ }^{a}$ As análises foram realizadas utilizando-se os procedimentos para amostras complexas ("svy”) do software Stata, versão 10.1.

Por se tratar de entrevista por telefone, o termo de consentimento livre e esclarecido foi substituído pelo consentimento verbal obtido por ocasião dos contatos telefônicos com os entrevistados. O VIGITEL foi aprovado pela Comissão de Ética em Pesquisa em Seres Humanos do Ministério da Saúde.

\section{RESULTADOS}

Entre os 9.038 participantes (5.973 mulheres e 3.065 homens), a média da idade foi de 69,7 anos (mín= 60 e máx=101 anos). O consumo de frutas e hortaliças inferior a cinco porções diárias $(89,0 \%)$ e a atividade física insuficiente no lazer $(86,3 \%)$ foram os comportamentos com prevalências mais altas, seguidos pela adição de sal à refeição $(59,4 \%)$, sobrepeso $(54,7 \%)$, consumo habitual de carnes com excesso de gordura $(23,0 \%)$, tabagismo $(11,5 \%)$ e consumo abusivo de bebidas alcoólicas (3,5\%). A prevalência de hipertensão auto-referida foi de $55,4 \%$, a prevalência de diabetes mellitus foi de $18,2 \%$ e a prevalência de dislipidemia foi de $35,7 \%$ (Tabela 1 ).

As correlações entre os comportamentos em saúde e sobrepeso variaram entre fraca e moderada (Tabela 2). De maneira geral, o tabagismo, o consumo abusivo de bebidas alcoólicas, o consumo insuficiente de frutas e

a Ministério da Saúde. Secretaria de Vigilância em Saúde. Secretaria de Gestão Estratégica e Participativa. Vigitel Brasil 2006: vigilância de fatores de risco e de proteção para doenças crônicas por inquérito telefônico. Brasília, DF; 2007. 
Tabela 1. Características dos idosos estudados. ${ }^{a}$ Brasil, 2006. ( $\left.N=9.038\right)$

\begin{tabular}{|c|c|}
\hline Característica & \% ou média (IC 95\%) \\
\hline \multicolumn{2}{|l|}{ Faixa etária (anos) } \\
\hline $60-69$ & $55,6(53,6 ; 57,7)$ \\
\hline $70-79$ & $31,8(30,0 ; 33,7)$ \\
\hline $80+$ & $12,5(11,2 ; 13,8)$ \\
\hline Média da idade & $69,7(69,4 ; 70,0)$ \\
\hline Sexo feminino & $59,6(57,5 ; 61,7)$ \\
\hline Tabagismo (fumante atual) & $11,5(10,1 ; 13,0)$ \\
\hline $\begin{array}{l}\text { Consumo abusivo de bebidas alcoólicas ( }>4 \text { doses [mulheres] ou }>5 \text { doses [homens] em uma } \\
\text { única ocasião nos últimos } 30 \text { dias) }\end{array}$ & $3,5(2,9 ; 4,2)$ \\
\hline Consumo insuficiente de frutas e hortaliças ( $<5$ porções diárias) & $89,0(87,8 ; 90,3)$ \\
\hline Consumo habitual de carnes com excesso de gordurab & $23,0(21,2 ; 24,9)$ \\
\hline Adição de sal à refeição pronta (sempre ou às vezes) & $59,4(57,3 ; 61,4)$ \\
\hline $\begin{array}{l}\text { Atividade física insuficiente no lazer (intensidade leve ou moderada por pelo menos } 30 \text { minutos } \\
<3 \text { dias na semana) }\end{array}$ & $86,3(84,8 ; 87,6)$ \\
\hline Sobrepeso (Índice de massa corporal > 25 kg/m²) & $54,7(52,4 ; 56,9)$ \\
\hline Diagnóstico médico prévio de hipertensão arterial & $55,4(53,3 ; 57,4)$ \\
\hline Diagnóstico médico prévio de diabetes mellitus & $18,2(16,6 ; 19,8)$ \\
\hline Diagnóstico médico prévio de dislipidemia & $37,5(33,8 ; 37,8)$ \\
\hline
\end{tabular}

a Ponderado para ajustar a distribuição sociodemográfica da amostra VIGITEL à distribuição da população adulta de cada cidade no Censo Demográfico de 2000 e considerando o peso populacional de cada cidade.

${ }^{\mathbf{b}}$ Consumo de carne vermelha ou frango sem pele sem remover a gordura visível do alimento.

hortaliças, o consumo habitual de carnes com excesso de gordura, a atividade física insuficiente no lazer e o sobrepeso estavam correlacionados, com poucas exceções. Por outro lado, a adição de sal à refeição foi o hábito que mostrou menos correlação com os demais, apresentando correlação somente com o consumo de carnes com gordura e com o sobrepeso.

Entre idosos que informaram ser hipertensos, a média do número de outros fatores de risco cardiovascular foi de 2,0 (IC 95\%: 2,01;2,10), incluindo-se nessa estimativa tabagismo, atividade física insuficiente no lazer, dislipidemia, sobrepeso e diabetes mellitus. Entre hipertensos, 3,1\% (IC 95\%: 2,4;3,9) não apresentavam outros fatores de risco, ao passo que 69,2\% (IC 95\%: $67,2 ; 71.3)$ apresentavam três ou mais desses fatores (Figura). Entre as mulheres, a média do número de fatores de risco foi superior $(2,16$; IC $95 \%$ : 2,11;2,22) à dos homens $(1,92$; IC 95\%: 1,84;1,99) $(<0,001)$.

$\mathrm{Na}$ Tabela 3 estão apresentadas as prevalências de fatores de risco entre idosos que informaram ser hipertensos em comparação aos que negaram ter essa condição. Entre hipertensos, foram observadas altas prevalências de atividades físicas insuficientes no lazer $(87,5 \%)$ e do consumo de frutas e hortaliças inferior a cinco porções diárias $(89,5 \%)$, seguidas pela adição de sal à refeição $(59,8 \%)$, consumo habitual de carnes gordurosas $(23,0 \%)$, tabagismo $(8,5 \%)$ e consumo abusivo de álcool (3,1\%). Exceto o tabagismo, as prevalências destes comportamentos não diferiram significativamente entre hipertensos e não hipertensos (RP ajustadas por idade e sexo variaram entre 0,99 [IC 95\%: 0,81;1,23] e 1,07 [IC 95\%: 0,95;1,20]). A prevalência da hipertensão auto-referida foi mais baixa entre fumantes do que entre não fumantes $(R P=0,75$; IC 95\%: 0,64;0,89) e mais alta entre idosos com sobrepeso em comparação àqueles com peso normal $(\mathrm{RP}=$ 1,37; IC 95\%: 1,25;1,49), assim como entre idosos com diagnóstico médico anterior para dislipidemia e diabetes, em comparação àqueles sem esses diagnósticos $(\mathrm{RP}=1,36$; IC 95\%: 1,26;1,36 e $(\mathrm{RP}=1,37$; IC $95 \%: 1,27 ; 1,37$, respectivamente).

As prevalências de fatores de risco entre hipertensos e não hipertensos segundo o sexo estão apresentadas na Tabela 4. As prevalências de todos os fatores de risco comportamentais foram semelhantes entre hipertensos e não hipertensos de ambos os sexos, exceto tabagismo. A prevalência de fumantes foi significativamente menor entre hipertensos em comparação a não hipertensos, tanto entre os homens quanto entre as mulheres. Por outro lado, as prevalências de sobrepeso, de diabetes mellitus e de dislipidemia auto-referidos foram maiores entre hipertensos de ambos os sexos. Não foram observadas interações significantes $(\mathrm{p} \geq$ $0,05)$ entre sexos e fatores de risco cardiovasculares na hipertensão auto-referida. 
Tabela 2. Coeficiente de correlação tetracórica entre comportamentos em saúde entre idosos. ${ }^{\text {a }}$ Brasil, 2006. (N=9.038)

\begin{tabular}{|c|c|c|c|c|c|c|}
\hline Fator de risco & Tabagismo & $\begin{array}{l}\text { Consumo } \\
\text { abusivo de } \\
\text { bebidas } \\
\text { alcoólicas }\end{array}$ & $\begin{array}{l}\text { Consumo } \\
\text { irregular } \\
\text { de frutas e } \\
\text { hortaliças }\end{array}$ & $\begin{array}{l}\text { Consumo } \\
\text { habitual de } \\
\text { carnes com } \\
\text { excesso de } \\
\text { gordura }\end{array}$ & $\begin{array}{l}\text { Adição de sal à } \\
\text { refeição pronta }\end{array}$ & $\begin{array}{c}\text { Atividade física } \\
\text { insuficiente no } \\
\text { lazer }\end{array}$ \\
\hline \multicolumn{7}{|l|}{ Tabagismo } \\
\hline $\begin{array}{l}\text { Consumo } \\
\text { abusivo de } \\
\text { bebidas } \\
\text { alcoólicas }\end{array}$ & $0,2626^{*}$ & & & & & \\
\hline $\begin{array}{l}\text { Consumo } \\
\text { insuficiente } \\
\text { de frutas e } \\
\text { hortaliças }\end{array}$ & $0,1368^{*}$ & 0,0123 & & & & \\
\hline $\begin{array}{l}\text { Consumo } \\
\text { habitual de } \\
\text { carnes com } \\
\text { excesso de } \\
\text { gordura }\end{array}$ & $0,2646^{*}$ & $0,2654^{*}$ & $0,1635^{*}$ & & & \\
\hline $\begin{array}{l}\text { Adição de sal à } \\
\text { refeição pronta }\end{array}$ & $-0,0477$ & $-0,0093$ & $-0,0074$ & $0,0506^{*}$ & & \\
\hline $\begin{array}{l}\text { Atividade física } \\
\text { insuficiente no } \\
\text { lazer }\end{array}$ & $0,1339^{*}$ & $-0,1369^{*}$ & $0,1660^{*}$ & $0,0970^{*}$ & $-0,0108$ & \\
\hline $\begin{array}{l}\text { Excesso de } \\
\text { peso }\end{array}$ & $-0,2196^{*}$ & $0,0969^{*}$ & $-0,0265^{*}$ & 0,0315 & 0,0434 * & $-0,0339$ \\
\hline
\end{tabular}

a Ponderado para ajustar a distribuição sociodemográfica da amostra VIGITEL à distribuição da população adulta de cada cidade no Censo Demográfico de 2000 e considerando o peso populacional de cada cidade.

${ }^{*} p<0,05$

\section{DISCUSSÃO}

Os resultados do presente trabalho, conduzido em uma grande amostra de idosos residentes em 27 cidades brasileiras, mostraram altas prevalências de fatores de risco comportamentais e de outros fatores de risco cardiovascular entre idosos hipertensos e não hipertensos. À exceção do tabagismo, as prevalências dos fatores de risco comportamentais foram semelhantes entre aqueles que informaram diagnóstico prévio para hipertensão e entre os que negaram essa condição. Isso sugere que os comportamentos prejudiciais à saúde persistiram mesmo após o diagnóstico da doença.

Estudos anteriores têm mostrado que a concomitância de fatores de risco cardiovascular predomina entre hipertensos. Nos Estados Unidos, no estudo de Framingham, 19\% dos hipertensos apresentaram hipertensão arterial não acompanhada de outros fatores de risco, ao passo que $30 \%$ apresentavam três ou mais desses fatores. ${ }^{12}$ Resultados semelhantes foram observados no Brasil entre participantes do estudo de Bambuí (MG). Entre hipertensos, 7\% apresentavam hipertensão não acompanhada de outros fatores de risco, enquanto $41 \%$ apresentavam três ou mais desses fatores. ${ }^{1}$ Esses estudos diferem da presente investigação porque foram conduzidos em adultos mais jovens ( $\geq 18$ anos) e foram baseados em medidas objetivas da pressão arterial, da dislipidemia e do IMC, além de informações sobre comportamentos em saúde. No presente trabalho, a aglomeração de fatores de risco cardiovascular foi mais alta: $3 \%$ dos hipertensos não apresentavam outros

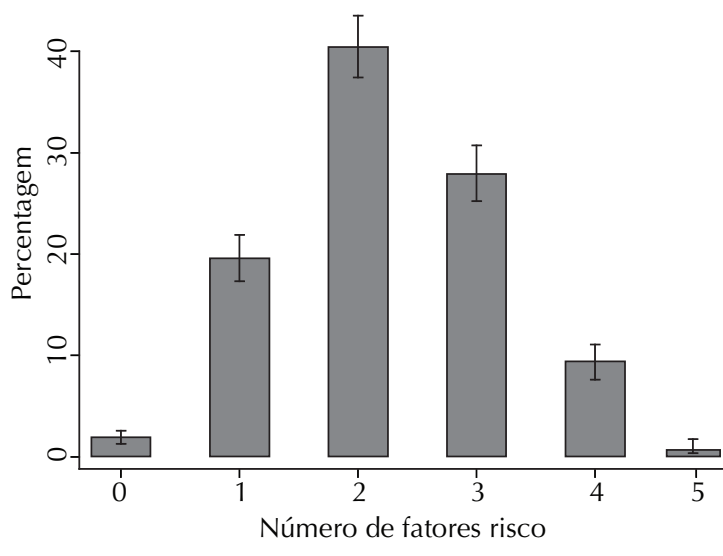

Figura. Número de fatores de risco cardiovascular (tabagismo, atividade física insuficiente no lazer, dislipidemia, sobrepeso e diabetes mellitus) entre idosos que informaram diagnóstico médico prévio para hipertensão arterial. Brasil, 2006. $(\mathrm{N}=9.038)$ 
Tabela 3. Associação entre fatores de risco cardiovascular e diagnóstico prévio de hipertensão arterial entre idosos. ${ }^{\mathbf{a}}$ Brasil, 2006. $(\mathrm{N}=9.038)$

\begin{tabular}{lccc}
\hline & \multicolumn{2}{c}{ Diagnóstico prévio de hipertensão arterial } & RP (IC 95\%) \\
\hline Fator de risco & Sim & Não & \% (IC 95\%) \\
\hline Tabagismo & $8,5(7,1 ; 10,3)$ & $15,1(12,7 ; 17,9)$ & $0,75(0,64 ; 0,89)$ \\
Consumo abusivo de bebidas alcoólicas & $3,1(2,3 ; 4,1)$ & $3,9(3,0 ; 5,1)$ & $0,99(0,81 ; 1,23)$ \\
Consumo insuficiente de frutas e hortaliças & $89,5(87,7 ; 90,2)$ & $88,4(86,3 ; 90,2)$ & $1,07(0,95 ; 1,21)$ \\
Consumo habitual de carnes com excesso de gordura & $23,0(21,2 ; 24,9)$ & $20,6(18,3 ; 23,1)$ & $0,92(0,83 ; 1,02)$ \\
Adição de sal à refeição pronta & $59,8(57,1 ; 62,5)$ & $58,8(55,7 ; 61,9)$ & $1,02(0,95 ; 1,10)$ \\
Atividade física insuficiente no lazer & $87,5(85,7 ; 89,1)$ & $84,7(82,2 ; 87,0)$ & $1,07(0,95 ; 1,20)$ \\
Sobrepeso & $62,4(59,5 ; 65,3)$ & $45,3(41,9 ; 48,8)$ & $1,37(1,25 ; 1,49)$ \\
Diagnóstico médico prévio para diabetes mellitus & $23,6(21,3 ; 26,1)$ & $11,5(9,7 ; 13,6)$ & $1,37(1,27 ; 1,47)$ \\
Diagnóstico médico prévio para dislipidemia & $43,7(41,0 ; 46,5)$ & $26,0(23,3 ; 28,8)$ & $1,36(1,26 ; 1,36)$ \\
\hline
\end{tabular}

a Ponderado para ajustar a distribuição sociodemográfica da amostra VIGITEL à distribuição da população adulta de cada cidade no Censo Demográfico de 2000 e considerando o peso populacional de cada cidade.

RP (IC 95\%): razões de prevalência ajustadas por idade e sexo estimadas pela regressão de Poisson (a classe de referência é não hipertenso).

fatores de risco e $69 \%$ apresentavam três ou mais fatores. Considerando-se que o risco cardiovascular aumenta progressivamente com o número de fatores de risco presentes, ${ }^{12}$ a distribuição desses fatores na população estudada caracteriza situação de maior risco para esses eventos.

A atividade física é o fator que prediz de forma mais consistente o envelhecimento saudável, como tem sido observado em diversos estudos de coorte de idosos. ${ }^{10,11,14,22,25, a}$ As atividades físicas reduzem a mortalidade cardiovascular, independentemente da pressão arterial e de outros fatores de risco. ${ }^{18}$ Adicionalmente, existem fortes evidências de que exercícios físicos reduzem a pressão arterial. ${ }^{5}$ Recomenda-se a prática de exercícios físicos de intensidade leve ou moderada para prevenir a hipertensão arterial e para o controle da hipertensão já instalada. ${ }^{18}$ No presente trabalho, a prevalência da atividade física insuficiente no lazer foi igualmente alta em hipertensos ( $88 \%$ ) e não hipertensos $(85 \%)$ de ambos os sexos. Altas prevalências de atividades físicas insuficientes no lazer são também observadas em outras populações (82\% entre idosos norte-americanos, por exemplo). ${ }^{a}$ Essas prevalências refletem a insuficiência de medidas para aumentar adequadamente essa prática na população.

A influência dos hábitos alimentares na morbimortalidade cardiovascular é amplamente reconhecida. Dietas ricas em frutas e hortaliças e com produtos com baixo teor de gorduras diminuem o risco cardiovascular, contribuem para a redução do peso e atuam diretamente na redução da pressão arterial. ${ }^{18, a}$ Como estratégia de prevenção primária e secundária, recomenda-se a ingestão diária de cinco ou mais porções de frutas e hortaliças, correspondendo a cerca de 400 g. ${ }^{18,28, a}$ No presente trabalho, o consumo inadequado desses alimentos foi um dos dois fatores comportamentais com mais alta prevalência entre hipertensos $(90 \%)$ e nãohipertensos (88\%). A melhora desse hábito alimentar não é uma tarefa trivial. Apesar das metas estabelecidas no documento Healthy People 2010 para aumentar o consumo de frutas e hortaliças, a prevalência de consumo insuficiente desses alimentos entre adultos norte-americanos não se modificou nos últimos dez anos. $^{2}$ No presente trabalho, o consumo habitual de carnes com excesso de gordura foi igualmente relatado por idosos hipertensos (23\%) e não hipertensos (21\%). Esse hábito foi o que apresentou maior disparidade entre os sexos, com prevalência duas vezes maior entre os homens do que as mulheres, diferença a ser considerada em políticas para a redução do hábito.

O consumo de sal no Brasil é alto, excedendo os limites máximos recomendados para a sua ingestão em todas as regiões do País e em todos os estratos de renda. ${ }^{24}$ A restrição de sal tem efeito anti-hipertensivo, ${ }^{7}$ sobretudo se combinada a outros hábitos alimentares saudáveis, e pode levar à redução da dose da medicação empregada para reduzir a pressão arterial. ${ }^{18} \mathrm{O}$ hábito de adicionar sal à refeição, ainda que esporadicamente, foi informado por mais da metade dos idosos hipertensos $(60 \%)$ e não-hipertensos $(59 \%)$ do presente estudo. Esse foi o único comportamento que não apresentou correlação com os demais (exceto consumo de carnes com gordura e sobrepeso). A definição do uso de sal adotada no presente trabalho foi limitada,

a U.S. Department of Health \& Human Services. Agency for Healthcare Research and Quality. U.S. Preventive Services Task Force (USPSTF). [citado 2005 Nov 13]. Disponível em: http://www.ahrq.gov 
Tabela 4. Associação entre fatores de risco cardiovascular e diagnóstico prévio de hipertensão arterial entre idosos, segundo sexo. ${ }^{a}$ Brasil, 2006. ( $\left.\mathrm{N}=9.038\right)$

\begin{tabular}{|c|c|c|c|c|c|c|}
\hline \multirow{3}{*}{ Fator de risco } & \multicolumn{3}{|c|}{ Masculino } & \multicolumn{3}{|c|}{ Feminino } \\
\hline & \multicolumn{2}{|c|}{$\begin{array}{l}\text { Diagnóstico prévio de } \\
\text { hipertensão arterial }\end{array}$} & \multirow[t]{2}{*}{ RP (IC 95\%) } & \multicolumn{2}{|c|}{$\begin{array}{l}\text { Diagnóstico prévio de } \\
\text { hipertensão arterial }\end{array}$} & \multirow[t]{2}{*}{ RP (IC 95\%) } \\
\hline & $\underset{\%(I C ~ 95 \%)}{\operatorname{Sim}}$ & $\begin{array}{c}\text { Não } \\
\%(\text { IC 95\%) }\end{array}$ & & $\underset{\text { Sim }}{\text { SiC } 95 \%)}$ & $\begin{array}{c}\text { Não } \\
\%(\text { (IC 95\%) }\end{array}$ & \\
\hline Tabagismo & $\begin{array}{c}12,9 \\
(9,8 ; 16,8)\end{array}$ & $\begin{array}{c}19,8 \\
(15,7 ; 24,7)\end{array}$ & $\begin{array}{c}0,76 \\
(0,59 ; 0,98)\end{array}$ & $\begin{array}{c}6,2 \\
(4,8 ; 8,0)\end{array}$ & $\begin{array}{c}11,1 \\
(8,7 ; 14,9)\end{array}$ & $\begin{array}{c}0,75 \\
(0,61 ; 0,91)\end{array}$ \\
\hline $\begin{array}{l}\text { Consumo abusivo } \\
\text { de bebidas alcoólicas }\end{array}$ & $\begin{array}{c}7,4 \\
(5,3 ; 10,1)\end{array}$ & $\begin{array}{c}7,0 \\
(5,1 ; 9,4)\end{array}$ & $\begin{array}{c}1,06 \\
(0,83 ; 1,36)\end{array}$ & $\begin{array}{c}0,8 \\
(0,5 ; 1,4)\end{array}$ & $\begin{array}{c}1,3 \\
(0,8 ; 2,1)\end{array}$ & $\begin{array}{c}0,83 \\
(0,58 ; 1,20)\end{array}$ \\
\hline $\begin{array}{l}\text { Consumo insuficiente } \\
\text { de frutas e hortaliças }\end{array}$ & $\begin{array}{c}90,9 \\
(87,8 ; 93,2)\end{array}$ & $\begin{array}{c}91,2 \\
(88,1 ; 93,5)\end{array}$ & $\begin{array}{c}1,00 \\
(0,78 ; 1,27)\end{array}$ & $\begin{array}{c}88,8 \\
(86,6 ; 90,7)\end{array}$ & $\begin{array}{c}86,0 \\
(82,9 ; 88,5)\end{array}$ & $\begin{array}{c}1,11 \\
(0,97 ; 1,28)\end{array}$ \\
\hline $\begin{array}{l}\text { Consumo habitual de } \\
\text { carnes com excesso de gordura }\end{array}$ & $\begin{array}{c}31,9 \\
(27,1 ; 37,1)\end{array}$ & $\begin{array}{c}37,7 \\
(32,8 ; 42,9)\end{array}$ & $\begin{array}{c}0,87 \\
(0,73 ; 1,03)\end{array}$ & $\begin{array}{c}14,6 \\
(12,4 ; 17,1)\end{array}$ & $\begin{array}{c}15,1 \\
(12,6 ; 18,0)\end{array}$ & $\begin{array}{c}0,98 \\
(0,88 ; 1,10)\end{array}$ \\
\hline Adição de sal à refeição pronta & $\begin{array}{c}61,7 \\
(56,7 ; 66,5)\end{array}$ & $\begin{array}{c}58,2 \\
(53,0 ; 63,3)\end{array}$ & $\begin{array}{c}1,07 \\
(0,92 ; 1,26)\end{array}$ & $\begin{array}{c}58,8 \\
(55,6 ; 62,0)\end{array}$ & $\begin{array}{c}59,4 \\
(55,6 ; 63,0)\end{array}$ & $\begin{array}{c}0,99 \\
(0,91 ; 1,07)\end{array}$ \\
\hline $\begin{array}{l}\text { Atividade física } \\
\text { insuficiente no lazer }\end{array}$ & $\begin{array}{c}83,4 \\
(78,3 ; 85,8)\end{array}$ & $\begin{array}{c}80,5 \\
(75,7 ; 84,5)\end{array}$ & $\begin{array}{c}1,07 \\
(0,87 ; 1,31)\end{array}$ & $\begin{array}{c}90,2 \\
(88,5 ; 91,7)\end{array}$ & $\begin{array}{c}88,6 \\
(86,3 ; 90,6)\end{array}$ & $\begin{array}{c}1,07 \\
(0,95 ; 1,21)\end{array}$ \\
\hline Sobrepeso & $\begin{array}{c}59,5 \\
(54,2 ; 64,5)\end{array}$ & $\begin{array}{c}43,5 \\
(38,3 ; 48,9)\end{array}$ & $\begin{array}{c}1,42 \\
(1,20 ; 1,66)\end{array}$ & $\begin{array}{c}64,4 \\
(61,0 ; 67,7)\end{array}$ & $\begin{array}{c}47,5 \\
(43,3 ; 51,8)\end{array}$ & $\begin{array}{c}1,33 \\
(1,21 ; 1,47)\end{array}$ \\
\hline $\begin{array}{l}\text { Diagnóstico médico } \\
\text { prévio para diabetes mellitus }\end{array}$ & $\begin{array}{c}20,2 \\
(16,5 ; 24,6)\end{array}$ & $\begin{array}{c}11,5 \\
(8,9 ; 14,8)\end{array}$ & $\begin{array}{c}1,37 \\
(1,16 ; 1,60)\end{array}$ & $\begin{array}{c}25,4 \\
(22,6 ; 28,4)\end{array}$ & $\begin{array}{c}11,6 \\
(9,2 ; 14,5)\end{array}$ & $\begin{array}{c}1,36 \\
(1,26 ; 1,48)\end{array}$ \\
\hline $\begin{array}{l}\text { Diagnóstico médico } \\
\text { prévio para dislipidemia }\end{array}$ & $\begin{array}{c}35,9 \\
(31,1 ; 41,0)\end{array}$ & $\begin{array}{c}18,8 \\
(15,0 ; 23,2)\end{array}$ & $\begin{array}{c}1,52 \\
(1,31 ; 1,76)\end{array}$ & $\begin{array}{c}47,9 \\
(44,7 ; 51,2)\end{array}$ & $\begin{array}{c}32,3 \\
(28,9 ; 36,0)\end{array}$ & $\begin{array}{c}1,29 \\
(1,19 ; 1,39)\end{array}$ \\
\hline
\end{tabular}

a Ponderado para ajustar a distribuição sociodemográfica da amostra VIGITEL à distribuição da população adulta de cada cidade no Censo Demográfico de 2000 e considerando o peso populacional de cada cidade.

RP (IC 95\%): razões de prevalência ajustadas por idade e estimadas pela regressão de Poisson (a classe de referência é não hipertenso).

não permitindo quantificar a ingestão da substância. Entretanto, a alta prevalência de pessoas hipertensas e não hipertensas que informaram adicionar sal à comida é uma indicação de preferência pelo sal.

A obesidade ou o excesso de peso têm sido descritos como fator de risco para o envelhecimento mal-sucedido em vários estudos. ${ }^{3,6,10,22,24} \mathrm{O}$ risco de morrer apresenta uma relação linear com o IMC. Pessoas com excesso de peso têm maior probabilidade de desenvolver hipertensão, diabetes do tipo 2 e fatores de risco para outras condições crônicas, tais como dislipidemia. A redução do peso corporal reduz a pressão arterial. ${ }^{18}$ No presente trabalho, a prevalência do sobrepeso foi significativamente maior entre idosos hipertensos $(62 \%)$ do que entre não hipertensos (45\%), como era de se esperar.

O tabagismo é a maior causa de mortalidade cardiovascular no mundo. ${ }^{10}$ Embora a cessação do tabagismo não reduza a pressão arterial, ${ }^{23} \mathrm{o}$ abandono do hábito é, provavelmente, a medida isolada mais efetiva para a redução do risco de doenças cardiovasculares. ${ }^{9,18} \mathrm{O}$ consumo abusivo de álcool está associado ao maior risco de acidente vascular cerebral, ${ }^{27}$ que representa a complicação mais grave da hipertensão arterial. ${ }^{17}$ Por essas razões, a cessação do tabagismo e a moderação do consumo de álcool são recomendadas, tanto para a prevenção primária, quanto para a prevenção secundária. No presente trabalho, o tabagismo foi o único comportamento com menor prevalência entre hipertensos (9\%) do que entre não-hipertensos (15\%). A prevalência do consumo abusivo de álcool foi semelhante nos dois grupos ( $3 \%$ e $4 \%$, respectivamente). Esse resultado é consistente com a hipótese de que o diagnóstico da hipertensão tenha levado à cessação do tabagismo. Resultados de um estudo recente corroboram essa hipótese, o qual mostrou associação entre tentativas de parar de fumar e história médica de doenças relacionadas ao cigarro. ${ }^{6}$

O presente estudo tem vantagens e limitações. A principal vantagem é a abrangência da base de dados utilizada, cobrindo 27 cidades brasileiras. Outra vantagem é a grande amostra de participantes idosos, viabilizando estimativas com boa precisão. Além disso, existem evidências de boa reprodutibilidade e acurácia adequada dos instrumentos utilizados no VIGITEL, ${ }^{20,21}$ assegurando a qualidade da informação. Por outro lado, a amostra de participantes do estudo é constituída por residentes em domicílio com linha telefônica fixa, sendo essa uma das limitações do estudo. Para contornar essa limitação, foram feitos ajustes na distribuição sociodemográfica da amostra VIGITEL à distribuição 
da população das áreas do estudo, ${ }^{a}$ porém, não se pode garantir que os comportamentos em saúde e a morbidade auto-referida dos participantes sejam semelhantes às daqueles que residem em domicílios sem telefone fixo. Outra limitação é o uso da hipertensão auto-referida como variável dependente do estudo, em detrimento de medidas objetivas dessa condição (medidas de pressão arterial e uso de anti-hipertensivos). Embora existam evidências de boa sensibilidade e especificidade da hipertensão auto-referida entre idosos participantes de outro estudo brasileiro, ${ }^{15}$ não se sabe se esses resultados são generalizáveis. Além disso, o diagnóstico da hipertensão arterial é influenciado pelo uso de serviços de saúde, ${ }^{15}$ o que pode explicar, pelo menos em parte, a sua maior prevalência entre mulheres. ${ }^{16}$ Esse conjunto de fatores pode ter contribuído para subestimar as prevalências da hipertensão e para reduzir as forças das associações encontradas, tornando nossas estimativas conservadoras. Entretanto, a prevalência de hipertensão arterial observada na população estudada (55\%) foi pouco menor que a observada em estudos baseados em medidas objetivas da pressão arterial em idosos, tanto no Brasil ${ }^{1}$ quanto na América Latina e Caribe. ${ }^{13}$

\section{REFERÊNCIAS}

1. Barreto SM, Passos VMA, Firmo JOA, Guerra HL, Vidigal PG, Lima-Costa MF. Hypertension and clustering of cardiovascular risk factors in a community in Southeast Brazil: the Bambuí health and ageing study. Arq Bras Cardiol. 2001;77(6):576-81. DOI:10.1590/S0066-782X2001001200008

2. Blanck HM, Gillespie C, Kimmons JE, Seymour JD, Serdula MK. Trends in fruit and vegetable consumption among U.S. men and women, 1994-2005. Prev Chronic Dis. 2008;5(2):A35.

3. Burke GL, Arnold AM, Bild DE, Cushman M, Fried LP, Newman A, et al. Factors associated with healthy aging: the cardiovascular health study. I Am Geriatr Soc. 2001;49(3):254-62. DOI:10.1046/j.15325415.2001.4930254.x

4. Chobonian AV, Bakris GL, Black HR, Cushman WC, Green LA, Izzo Jr JL, et al. The Seventh Report of the Joint National Commitee on Prevention, Detection, Evaluation, and Treatment of High Blood Pressure: the JNC 7 Report. JAMA. 2003;289(19):2560-72.

5. Cornelissen VA, Fagard RH. Effect of resistance training on resting blood pressure: a meta-analysis of randomized controlled trials. J Hypertens. 2005;23(2):25-9.

6. Davila EP, Zhao W, Byrne M, Webb M, Huang Y, Arheat $\mathrm{K}$, et al. Correlates of smoking quit attempts: Florida Tobacco Callback Survey, 2007. Tob Induc Dis. 2009;5:10. DOI:10.1186/1617-9625-5-10.
Os comportamentos em saúde têm início cedo na vida e os seus efeitos são acumulativos. ${ }^{26,29, b}$ Dessa forma, embora as intervenções para melhora desses comportamentos tenham maior impacto quando voltadas para as populações jovens e de meia idade, as mudanças em idades mais avançadas trazem evidentes benefícios para a saúde. ${ }^{19}$ Além disso, como a morbidade e a mortalidade aumentam com a idade, e a hipertensão é emblemática dessa situação, os riscos atribuíveis associados a esses comportamentos são altos nas faixas etárias superiores. A adoção de hábitos saudáveis como medida de prevenção primária é amplamente reconhecida. Entretanto, a adoção de hábitos saudáveis entre hipertensos, como medida de prevenção secundária, tem recebido pouca atenção. Os resultados do presente trabalho revelam a magnitude do problema, reforçando a necessidade de estratégias voltadas para a adoção de hábitos saudáveis entre idosos hipertensos. Abordagens voltadas para múltiplos fatores de risco podem ser mais efetivas, uma vez que vários fatores comportamentais estavam correlacionados.

7. Dickinson HO, Mason JM, Nicolson DJ, Campbell F, Beyer FR, Cook JV, et al. Lifestyle interventions to reduce raised blood pressure: a systematic review of randomized controlled trials. J Hypertens. 2006;24(2):215-33.

8. Ezzati M, Lopez AD, Rodgers A, Vander Hoorn S, Murray CJ. Comparative Risk Assessment Collaborating Group. Selected major risk factors and global and regional burden of disease. Lancet. 2002;360(9343):1347-60. DOI:10.1016/S01406736(02)11403-6

9. Ezzati M, Henley SJ, Thun MJ, Lopez AD. Role of smoking in global and regional cardiovascular mortality. Circulation. 2005;112(4):456-8. DOI:10.1161/CIRCULATIONAHA. 104.521708

10. Guralnik JM, Kaplan GA. Predictors of healthy aging: prospective evidence from the Alameda County study. Am J Public Health. 1989;79(2):703-8. DOI:10.2105/ AJPH.79.6.703

11. Haveman-Nies A, de Groot LC, van Staveren WA. Dietary quality, lifestyle factors and healthy ageing in Europe: the SENECA study. Age Ageing. 2003;32(4):427-34. DOI:10.1093/ageing/32.4.427

12. Kannel WB. Risk stratification in hypertension: new insights from the Framingham Study. Am J Hypertens. 2000;13(1 Pt 2):3S-10S. DOI:10.1016/S08957061(99)00252-6

\footnotetext{
${ }^{a}$ Ministério da Saúde. Secretaria de Vigilância em Saúde. Secretaria de Gestão Estratégica e Participativa. Vigitel Brasil 2006: vigilância de fatores de risco e de proteção para doenças crônicas por inquérito telefônico. Brasília, DF; 2007.

${ }^{\mathrm{b}}$ U.S. Department of Health \& Human Services. Agency for Healthcare Research and Quality. U.S. Preventive Services Task Force (USPSTF). [citado 2005 Nov 13]. Disponível em: http://www.ahrq.gov
} 
13. Kearney PM, Whelton M, Reynolds K, Muntner P, Whelton PK, He J. Global burden of hypertension: analysis of worldwide data. Lancet. 2005;365(9455): 217-223.

14. Leveille SG, Guaralnik J, Ferrucci L, Langois JA. Aging successfully until death in old age: opportunities for increasing active life expectancy. Am J Epidemiol. 1999;149(7):654-64.

15. Lima-Costa MF, Peixoto SV, Firmo JO. Validade da hipertensão auto referida e seus determinantes (Projeto Bambuí). Rev Saude Publica. 2004;38(5):637-42. DOI:10.1590/S0034-89102004000500004

16. Lima-Costa MF, Loyola Filho Al, Matos DL. Tendências nas condições de saúde e uso de serviços de saúde entre idosos brasileiros: um estudo baseado na Pesquisa Nacional por Amostra de Domicílios (1998 e 2003). Cad Saude Publica. 2007;23(10):2467-78. DOI:10.1590/S0102-311X2007001000021

17. MacMahon S, Peto R, Cutler J, Collins R, Sorlie $\mathrm{P}$, Neaton J, et al. Blood pressure stroke coronary heart disease. Part 1 prolonged differences in blood pressure: prospective observational studies corrected for the regression dilution bias. Lancet. 1990:335(8692):765-74. DOI:10.1016/01406736(90)90878-9

18. Mancia G, De Backer G, Dominiczak A, Cifkova R, Fagard R, Germano G, et al. 2007 Guidelines for the Management of Arterial Hypertension: The Task Force for the Management of Arterial Hypertension of the European Society of Hypertension (ESH) and the European Society of Cardiology (ESC). Eur Heart ). 2007;28(12):1462-536.

19. Minker M, Schauffler H, Clements-Nolle K. Health promotion for older Americans in the $21^{\text {st }}$ century. Am J Health Promot. 2000;14(6):371-9.

20. Monteiro CA, Florindo AA, Claro RM, Moura EC. Validade de indicadores de atividade física e sedentarismo obtidos por inquérito telefônico. Rev Saude Publica. 2008;42(4):575-81. DOI: 10.1590/ S0034-89102008000400001

21. Monteiro CA, Moura EC, Jaime PC, Claro RM. Validade de indicadores do consumo de alimentos e bebidas obtidos por inquérito telefônico. Rev Saude Publica. 2008;42(4):582-9. DOI: 10.1590/S003489102008000400002

22. Newman AB, Arnold AM, Naydeck BL, Fried LP, Burke GL, Enright $P$, et al. "Successful aging": effect of subclinical cardiovascular disease. Arch Intern Med. 2003;163(19):2315-22. DOI:10.1001/ archinte.163.19.2315

23. Omvik P. How smoking affects blood pressure. Blood Press. 1996;5(2):71-7.

24. Sarno F, Claro RM, Levy RB, Bandoni DH, Ferreira SRG, Monteiro CA. Estimativa de consumo de sódio pela população brasileira, 2002-2003. Rev Saude Publica. 2009;43(2):219-225. DOI: 10.1590/S003489102009005000002

25. Strawbridge WJ, Cohen RD, Shema SJ, Kaplan G. Successful aging: predictors and associated activities. Am J Epidemiol. 1996;144(2):135-41.

26. Vita AJ, Terry RB, Hubert HB, Fries JF. Aging, health risks, and cumulative disability. $N$ Engl Med J. 1998;338(15):1035-41. DOI:10.1056/ NEJM199804093381506

27. Wannamethee SG, Shaper AG. Patterns of alcohol intake and risk of stroke in middle-aged British men. Stroke.1996;27:1033-9.

28. World Health Organization. Diet, Nutrition and the Prevention of Chronic Diseases. Report of a Joint WHO/FAO Expert Consultation. Geneva: WHO;2003 (WHO Technical Report Series 916).

29. World Health Organization. Active ageing: a police framework. Geneva; 2003.

MF Lima-Costa e CC Cesar foram apoiadas pelo Conselho Nacional de Desenvolvimento Científico e Tecnológico (CNPq 305227/206-8 e 307715/2008-6, respectivamente; bolsa produtividade em pesquisa).

Este estudo foi parcialmente desenvolvido pelo Núcleo de Estudos em Saúde Pública e Envelhecimento da Fundação Oswaldo Cruz e da Universidade Federal de Minas Gerais, Centro Colaborador da Secretaria de Vigilância em Saúde do Ministério da Saúde em Saúde do Idoso e Epidemiologia do Envelhecimento.

Artigo submetido ao processo de julgamento por pares adotado para qualquer outro manuscrito submetido a este periódico, com anonimato garantido entre autores e revisores. Editores e revisores declaram não haver conflito de interesses que pudesse afetar o processo de julgamento do artigo.

Os autores declaram não haver conflito de interesses. 